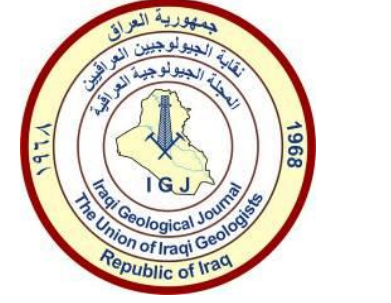

Iraqi Geological Journal

Journal homepage: https://www.igj-iraq.org

\title{
Geochemical and Clay Mineralogical Characteristics of the Black Shale and Constrains on Diagenesis and Maturation, Chia Gara Formation, Iraqi Kurdistan Region, Iraq
}

\author{
Faraj H. Tobia ${ }^{1, *}$ and Rezhin K. Mustafa ${ }^{1}$ \\ Department of Geology, College of Science, Salahaddin University, Erbil, Iraq \\ Correspondence: farajabba58@gmail.com
}

Received:
5 September 2021
Accepted:
19 November 2021
Published:
28 February 2022

\section{Abstract}

Black shale of Tithonian (Late Jurassic)-Berriasian (Early Cretaceous) Chia Gara Formation have been investigated by clay minerals and geochemistry (total organic carbon, major and trace elements) to determine the chemical associations and the relation between their diagenesis and maturity. The X-ray diffraction data show that kaolinite and illite are predominant clay minerals. The samples show low illite crystallinity index $\left(0.56^{\circ} \Delta 2 \theta\right)$, and most of the illite-smectite mixed layers are altered to the illite mineral. Analyzed black shales are recognized by high total organic carbon (TOC) content (1.47$5.87 \mathrm{wt} \%)$ and rich in $\mathrm{SiO}_{2}(33.19 \%)$, followed by $\mathrm{CaO}(20.54 \%)$ and $\mathrm{Al}_{2} \mathrm{O}_{3}(12.08 \%)$. Comparison between the obtained data and the Post Archean Australian Shale values indicate that $\mathrm{CaO}, \mathrm{Sr}, \mathrm{U}, \mathrm{V}, \mathrm{Ni}, \mathrm{Zn}$ and Mo were present in higher concentrations; $\mathrm{P}_{2} \mathrm{O}_{5}$, $\mathrm{Nb}$ and $\mathrm{Hf}$ were nearly similar, while $\mathrm{Na}_{2} \mathrm{O}, \mathrm{MnO}, \mathrm{Rb}$ and $\mathrm{Ba}$ was much lower. Also, the behavior of the trace elements can be inferred from the enrichment factor. The studied black shales are enriched in $\mathrm{Zn}, \mathrm{U}, \mathrm{V}, \mathrm{Ni}$ and Mo. Correlation between elements predict their association and origin. $\mathrm{U}, \mathrm{V}, \mathrm{Co}, \mathrm{Ni}, \mathrm{Cu}, \mathrm{Zn}$ and $\mathrm{Mo}$ are related to the phosphate minerals, also the organic matter played a part in the enrichment of $\mathrm{V}$ and $\mathrm{Ni}$ elements. $\mathrm{Al}_{2} \mathrm{O}_{3}$ significantly correlates with $\mathrm{Fe}_{2} \mathrm{O}_{3}, \mathrm{MnO}, \mathrm{TiO}_{2}, \mathrm{Sc}, \mathrm{Hf}, \mathrm{Nb}, \mathrm{Zr}, \mathrm{Th}, \mathrm{Ba}, \mathrm{Rb}$ in addition to REE indicating their associations with clay minerals. Dependent upon the predominance of illite clay mineral and illite crystallinity index, in addition to the $T_{\max }$ $\left(426\right.$ and $442^{\circ} \mathrm{C}$ ), the plurality of the studied shales is over mature and anchi-metamorphic. The shales are related to the deep diagenetic zone with a paleo-temperature between 150 and $200^{\circ} \mathrm{C}$.

Keywords: Black shale; Chia Gara Formation; Organic matter; Diagenesis; Maturation

\section{Introduction}

Black shales are dark-colored, usually laminated, organic-rich sediments which are related with Upper Jurassic-Lower Cretaceous claystone, clayey sandstone and limestone that are enriched in organic carbon (Wignall, 1994; Pancost et al. 2004). Majority shales have $1 \%$ or more OC, and the range 2-10 $\%$ is common, in addition few shales contain more than $20 \%$ OC (Tourtelot, 1979). Sufficient organic carbon is $0.3 \% \mathrm{wt}$. for the source rock potential of the oil-producing rocks of marine origin (Kuşcu et al. 2016). The researchers payed attention to the black shale due to the economic utility due to their

DOI: 10.46717/igj.55.1B.3Ms-2022-02-19 
potential of the hydrocarbon (regards as source rock) and likewise are regarded as host rocks for metal concentrations (Wignall, 1994; Schultz, 2004). Schultz (2004) pointed out some of these black shales are mostly enriched in $\mathrm{V}, \mathrm{U}, \mathrm{Mo}, \mathrm{Zn}, \mathrm{Ni}$, and $\mathrm{Cu}$. Tourtelot (1979) suggests that geological processes factors controlled the accumulation of the black shale not geological settings. Geological processes include the accumulation of the shale and the diagenesis that increased with the burial depth. Anthogenesis processes such as transformation and recrystallization, impact mineralogy, geochemistry and texture of the features of the rocks. The changes on the layers and interlayers of the clays increase due to the replacement in the octahedral and tetrahedral sheet during the conversion and successive ordering of the clay minerals, together with increasing degree of diagenesis (Peacor, 1992; Bozkaya and Yalcin, 2013). Trace and REE composition of the clay minerals have been employed to study the provenance and sedimentary processes (Condie, 1991; Tobia et al. 2018) and to determine the diagenetic grades (Ohr et al. 1994).

The evaluating of the hydrocarbon potential, in addition to the sedimentology, petrology and organic geochemistry of Chia Gara shale where studied intensively (Mohialdeen et al. 2013; Sherwani and Edilbi, 2019). In these studies, the trace elements and REEs received little attention (Mustafa and Tobia, 2020), although they are important in deciphering the depositional environment, weathering intensity and influence of other geological processes. Chia Gara Formation was firstly described by Wetzel (Bellen et al. 1959) in the High Folded Zone within Chia Gara anticline (Fig. 1).

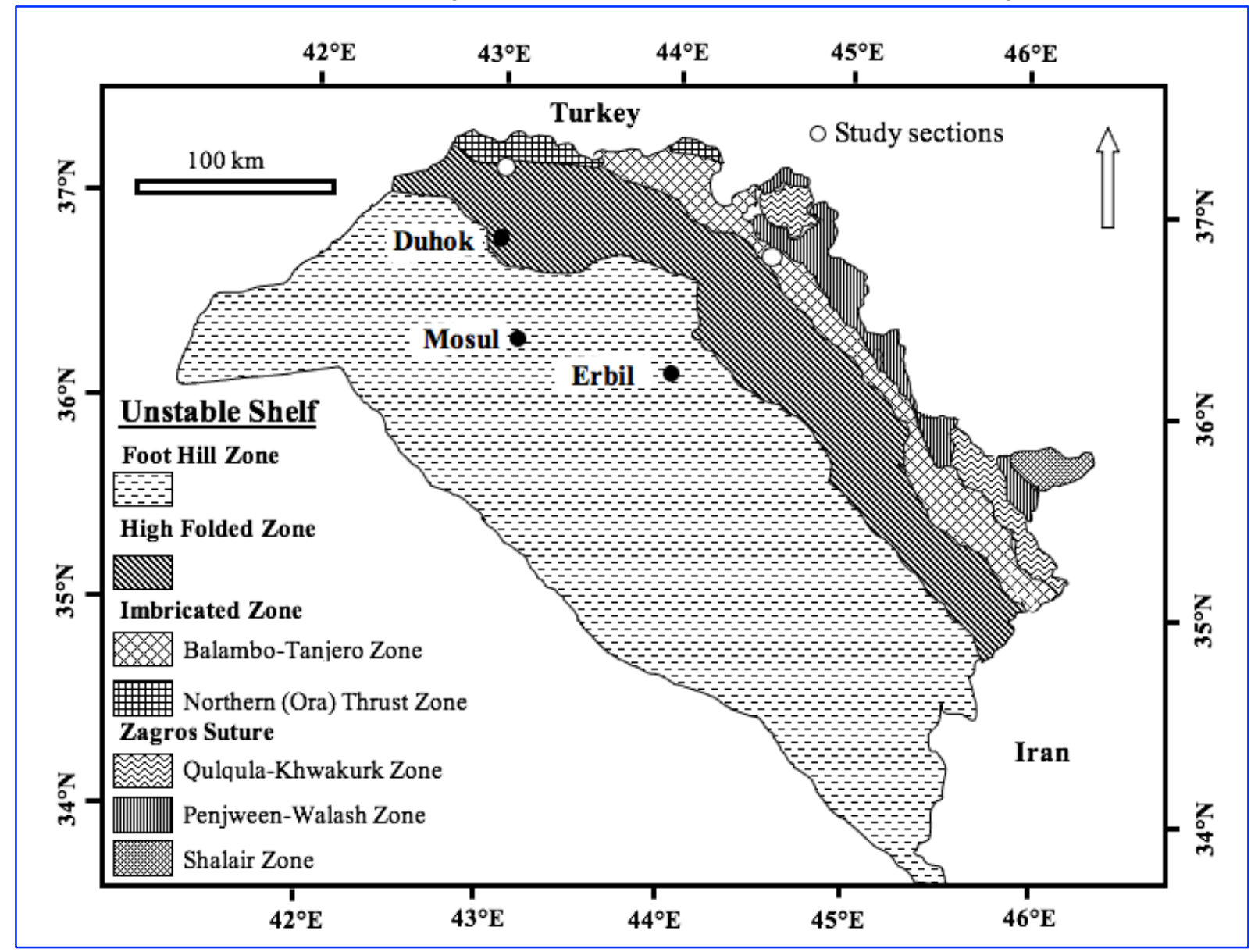

Fig. 1. Location and structural map of the studied area, Kurdistan Region, Iraq (after Jassim and Goff, 2006)

The thickness in the type section is up to $230 \mathrm{~m}$ of thin beds of limestone and calcareous shale with abundance of ammonite faunas, in addition to phacoid beds and bullion zone at the base. The formation 
was conformably underlain by Barsarin Formation. However, Spath (1950) and Bellen et al. (1959) suggested that there is a stratigraphic break between Chia Gara and Barsarin formations. In type locality, the contact between Chia Gara and Garagu formations is conformable. However, in the Northern Thrust Zone, the upper contact is locally erosional; in this area either the Garagu or the Campanian Hadiena Formation overlies the Chia Gara Formation. This study is conducted to investigate the chemical associations with the clay minerals, and inquire the relationship between diagenesis and maturation of the black shale.

\section{Geological setting}

During Jurassic up to Late Cretaceous, slow subsidence joining with fluctuation of sea level cause creation of shallow, large intra-shelf basins on the Arabian Plate and passive margins of the Neo-Tethys Ocean (Murris, 1980; Al-Sharhan and Nairn, 1997). Tectonically, the studied formation can be regarded as a part of the megasequence AP8 (149 $\pm 49 \mathrm{Ma})$; and there is a regional unconformity on Arabian Plate (Mid Tithonian) that denotes to a boundary between AP7 and AP8 Megasequences. This could be an unconformity that reflect the spreading of oceanic floor through opening of the Southern Neo- Tethys Ocean and cause a drifting of a narrow microcontinent; and a new margin was formed along the northeast margin of the Arabian Plate (Fig. 2).

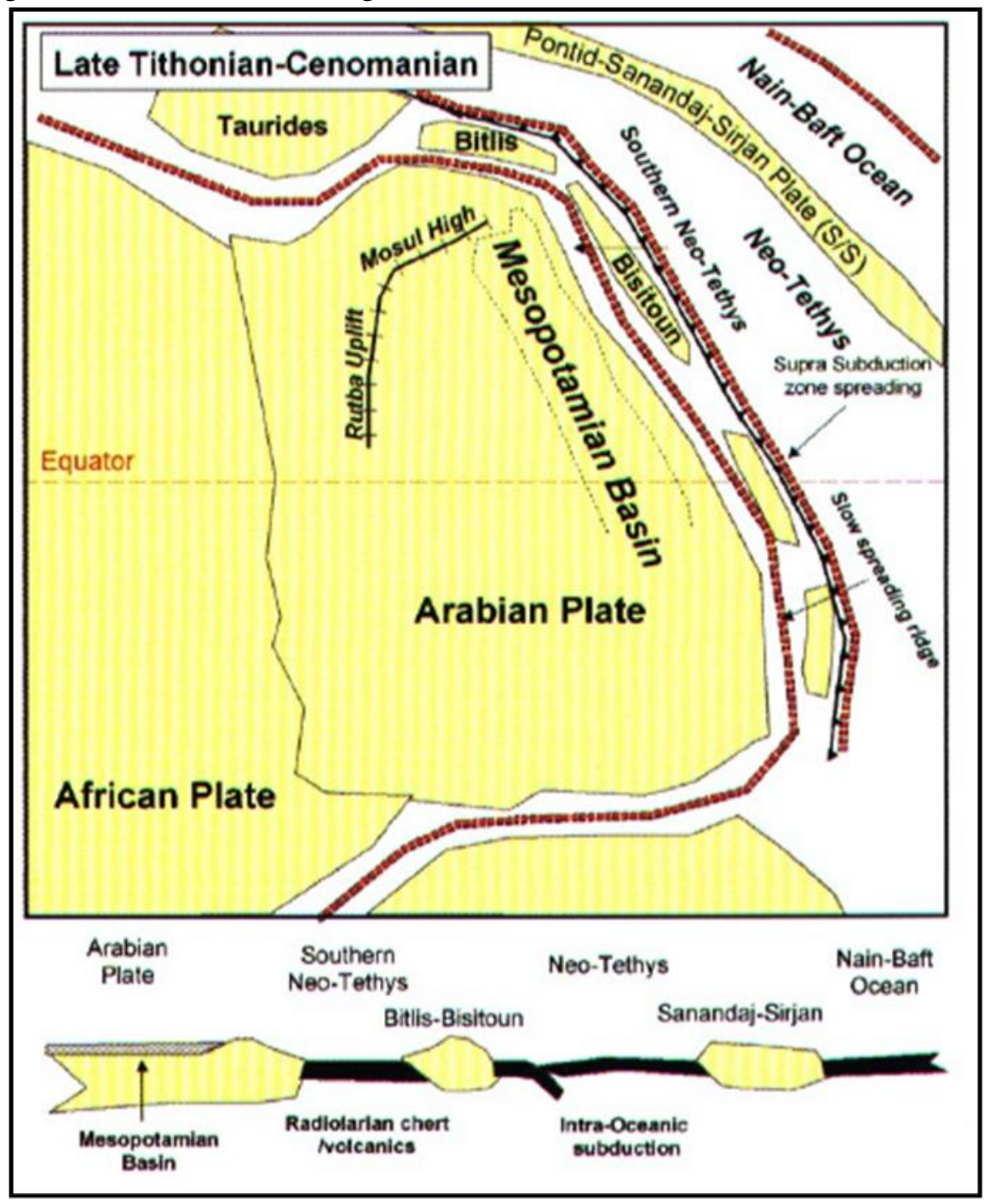

Fig. 2. Late Tithonian-Cenomanian geodynamic development of the Arabian Plate (after Jassim and Goff 2006). 
The formation is comprised of limestone and black shale enriched in organic matter and possibly depict an important source rock in Kurdistan Region (Odisho and Othman, 1992; Al-Beyati, 1998). This source rock is thermally mature, between early mature and peak oil generation window (Hakimi et al. 2017). The two studied sections are located within Thrust and High Folded Zones at the northeastern of the Arabian Plate margin (Fig. 1). Chia Gara Formation is restricted to the Unstable Shelf, toward SW gradually passes to the Makhul Formation in the Foothill Zone, and was deposited in Late Jurassic-Early Cretaceous as global separation and expansion time within the deep outer shelf of the Arabian Plate margins (Numan, 1997). Based on the fossil contents, the formation reveals a Mid Tithonian-Berriasian age (Jassim and Goff, 2006). It has been interpreted as a suboxic to anoxic conditions deposited under deep marine environment (Mohialdeen et al. 2013; Mustafa and Tobia, 2020), during High System Track and Transgression System Track stages (Sharland et al. 2001). The formation has high sedimentation rates involve shorter residence times, and rapid burial of OC during the diagenetic stage (Mohialdeen et al. 2013).

\section{Materials and Methods}

A total of 15 outcrop black shale samples were collected from the Chia Gara Formation ( 9 samples from Barsarin section at Imbricated Zone, and 6 samples from Banik section at Northern Thrust Zone; Fig. 1). The detailed sampling location and stratigraphic succession are presented in Fig. 3. A Bruker D2 Phaser X-ray generator with Ni-filtered $\mathrm{Cu} \mathrm{K \alpha}$ radiation, set at $40 \mathrm{kV}$ and $40 \mathrm{~mA}$, that is available at the Department of Geology/ University of Baghdad/ Iraq, used to examine the clay fractions $(<2 \mu \mathrm{m})$ and bulk samples. The clay fractions (after mounted on glass slides) were scanned at a range from $2^{\circ}$ to $20^{\circ} 2 \theta$ by a rotating holder, and the bulk samples from $3^{\circ}$ to $65^{\circ} 2 \theta$. For qualitative analysis of the clay minerals (15 samples), the samples were X-rayed under three conditions: air-dried, treated with ethylene glycol at $60^{\circ} \mathrm{C}$ for 12 hours, and heated to $550^{\circ} \mathrm{C}$ for two hours (Hardy and Tucker,1988). Minerals were distinguished by their characteristic reflections according to Moore and Reynolds (1997). Bulk mineralogy for four clay fraction air dried samples were studied with random orientation.

Geochemically, the samples were pulverized to $<200$ mesh in the agate mortar. The major oxides $\left(\mathrm{Al}_{2} \mathrm{O}_{3}, \mathrm{Fe}_{2} \mathrm{O}_{3}, \mathrm{SiO}_{2}, \mathrm{MgO}, \mathrm{CaO}, \mathrm{K}_{2} \mathrm{O}, \mathrm{Na}_{2} \mathrm{O}, \mathrm{MnO}, \mathrm{TiO}_{2}\right.$ in addition to $\left.\mathrm{P}_{2} \mathrm{O}_{5}\right)$ where determined by ICPAES under ME-ICP06 code for analysis. The trace ( $\mathrm{Sc}, \mathrm{Cr}, \mathrm{Ni}, \mathrm{Mo}, \mathrm{Zn}, \mathrm{V}, \mathrm{Co}, \mathrm{Rb}, \mathrm{Ba}, \mathrm{Sr}, \mathrm{U}, \mathrm{Th}, \mathrm{Y}$, $\mathrm{Hf}, \mathrm{Nb}$ and $\mathrm{Zr}$ ) and rare earth elements were determined by ICP-MS under ME-MS81 code at ALS laboratory (Spain). LOI data was specified by ignition of the samples at $100^{\circ} \mathrm{C}$ for $2 \mathrm{~h}$, after removing the moisture (at $100^{\circ} \mathrm{C}$ for $24 \mathrm{~h}$ ). TOC was determined under code C-IR06a by Leco furnace after leaching the carbonate with 25\% HCl. International standards AMIS0304 and OREAS-121 were utilized as references. The replication of the analyzes of the samples imply that the error for major oxides is better than 3\%, while the precision for other elements is between 1 and $10 \%$. Dependent upon the mentioned standards, precision and accuracy for the elements Mo, $\mathrm{Zn}, \mathrm{Nb}, \mathrm{Ni}, \mathrm{Ce}, \mathrm{Sm}, \mathrm{Pr}, \mathrm{Tb}, \mathrm{Eu}$ and $\mathrm{Lu}$ were within $\pm 2 \%$; while for $\mathrm{Ba}, \mathrm{Sr}, \mathrm{U}, \mathrm{Th}, \mathrm{Cr}, \mathrm{V}, \mathrm{Zr}, \mathrm{Cu}, \mathrm{Co}, \mathrm{Y}, \mathrm{Ho}, \mathrm{Dy}, \mathrm{Tm}$ and Er were \pm 5 ; and for Sc, La, Hf, Gd and Nd were \pm 10 .

\section{Results}

\subsection{Mineralogy}

The X-ray diffraction data shows kaolinite and illite as predominant minerals in the clay fraction of the black shale from Chia Gara Formation (Fig. 4). There is a large variation in the kaolinite and illite content of two studied sections, ranges between 0 and $100 \%$, with an average value $59.20 \%$ and $35.78 \%$ respectively (Table 1). 


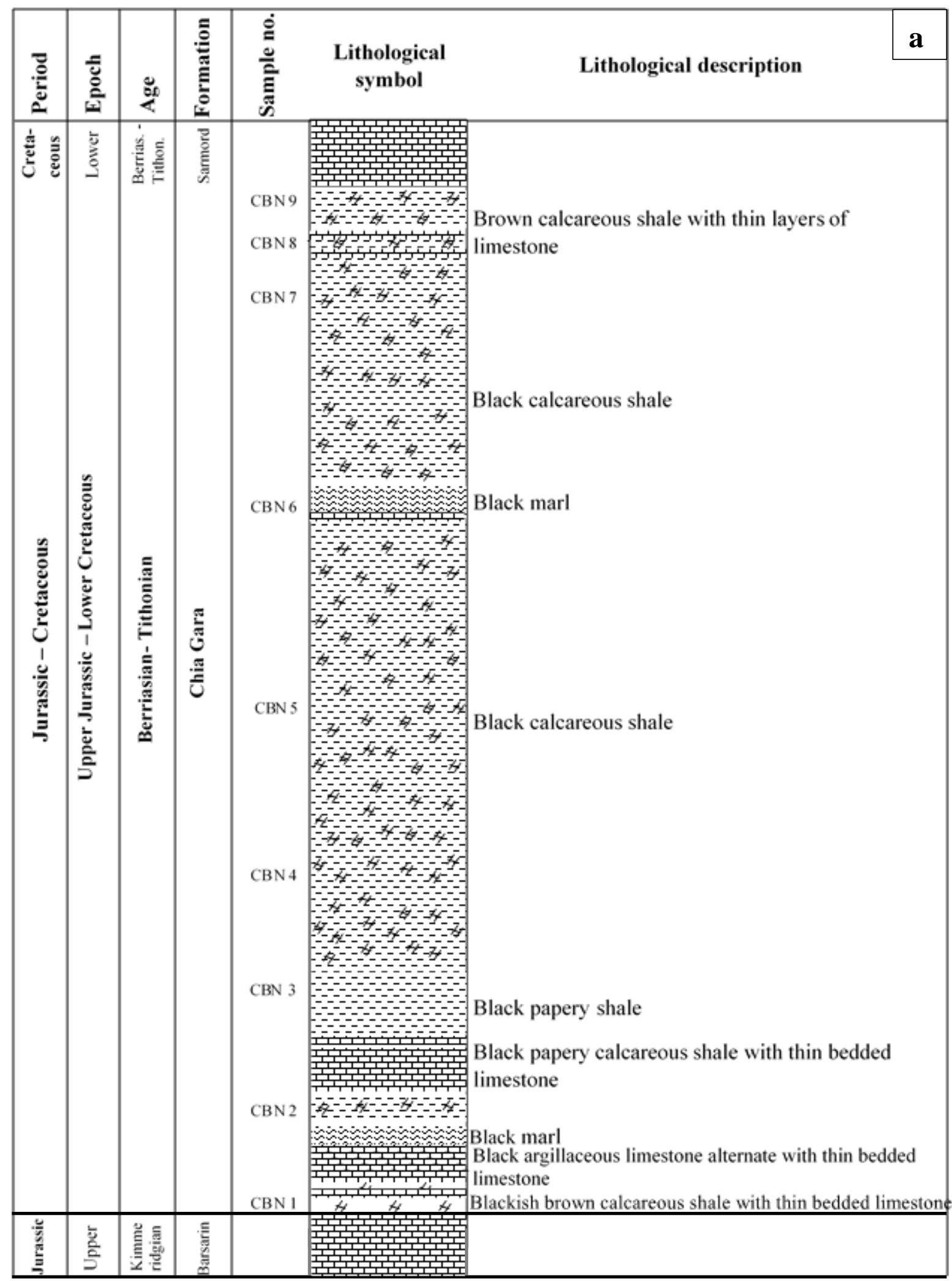

Fig. 3a. Columnar section of the Chia Gara Formation, Barsarin section

Scale 1: 1100; Thickness $=192 \mathrm{~m}$ 


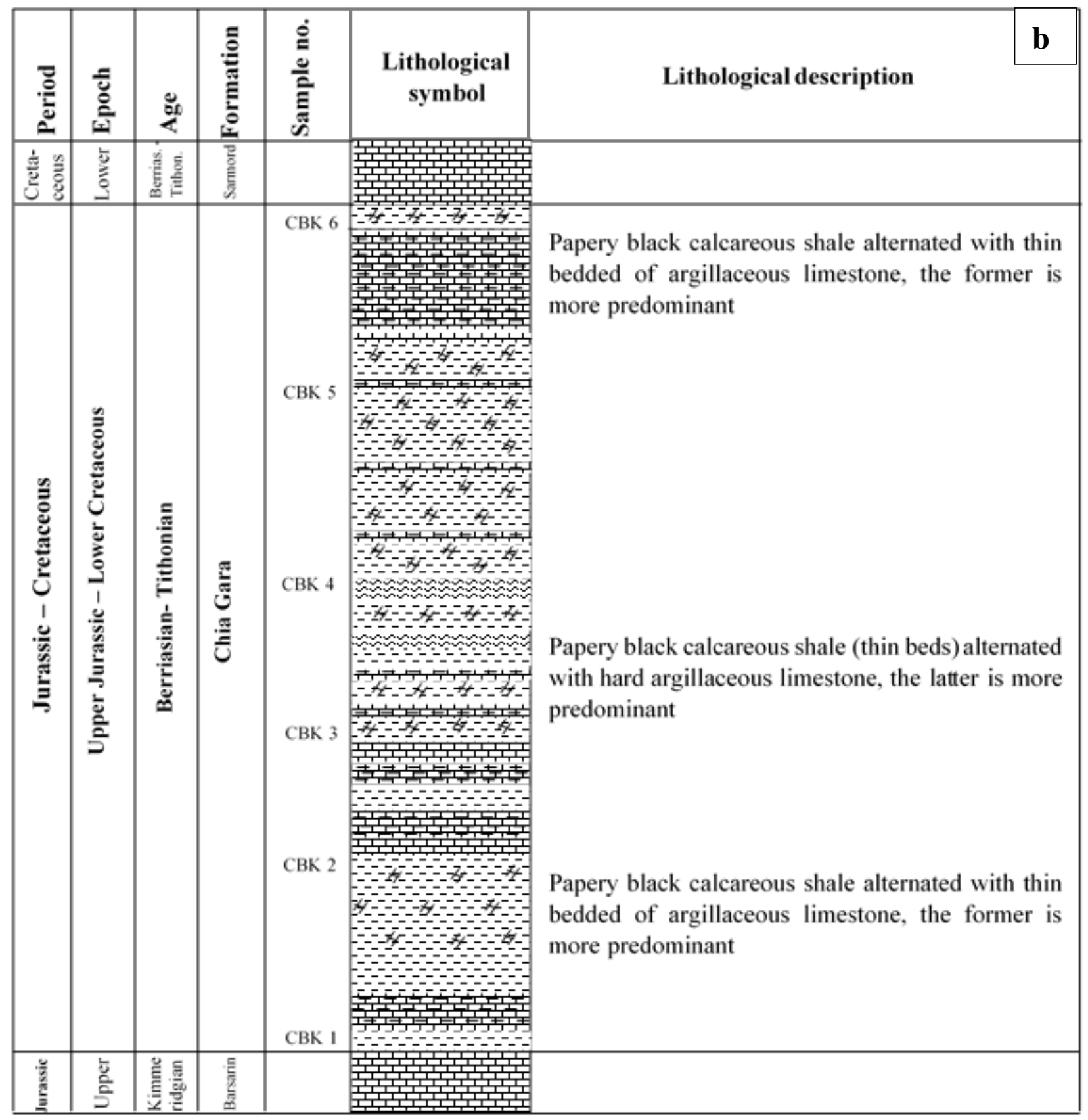

Fig. 3b. Columnar section of Chia Gara Formation, Banik section

Scale 1: 460; Thickness $=57.3 \mathrm{~m}$

The crystallinity index for both minerals have been calculated from the peak of each mineral. For kaolinite was determined by using the ratio of the measured width at the half-height of the $7 \AA$ peak for the air-dried state. For illite the crystallinity index (Kubler index) was estimated by measuring the peak width at half-height for the $10 \AA$ peak (Kubler, 1964; Segonzac, 1969). The crystallinity index of kaolinite ranges between 0.048 -0.5 with an average 0.10 ; and for illite (Kubler index) shows a low value varies between 0.37 to $0.80^{\circ} \Delta 2 \theta$, with an average $0.56^{\circ} \Delta 2 \theta$ (Table 1). Significant negative correlation was recorded between kaolinite and illite contents $(\mathrm{r}=0.862, \mathrm{n}=15$, at 0.01 level). The former positively correlate with $\mathrm{Zr}, \mathrm{Nb}$ and $\mathrm{Hf}(0.701,0.657$ and 0.689 , respectively, $\mathrm{n}=15)$ as shown in Table 2. 


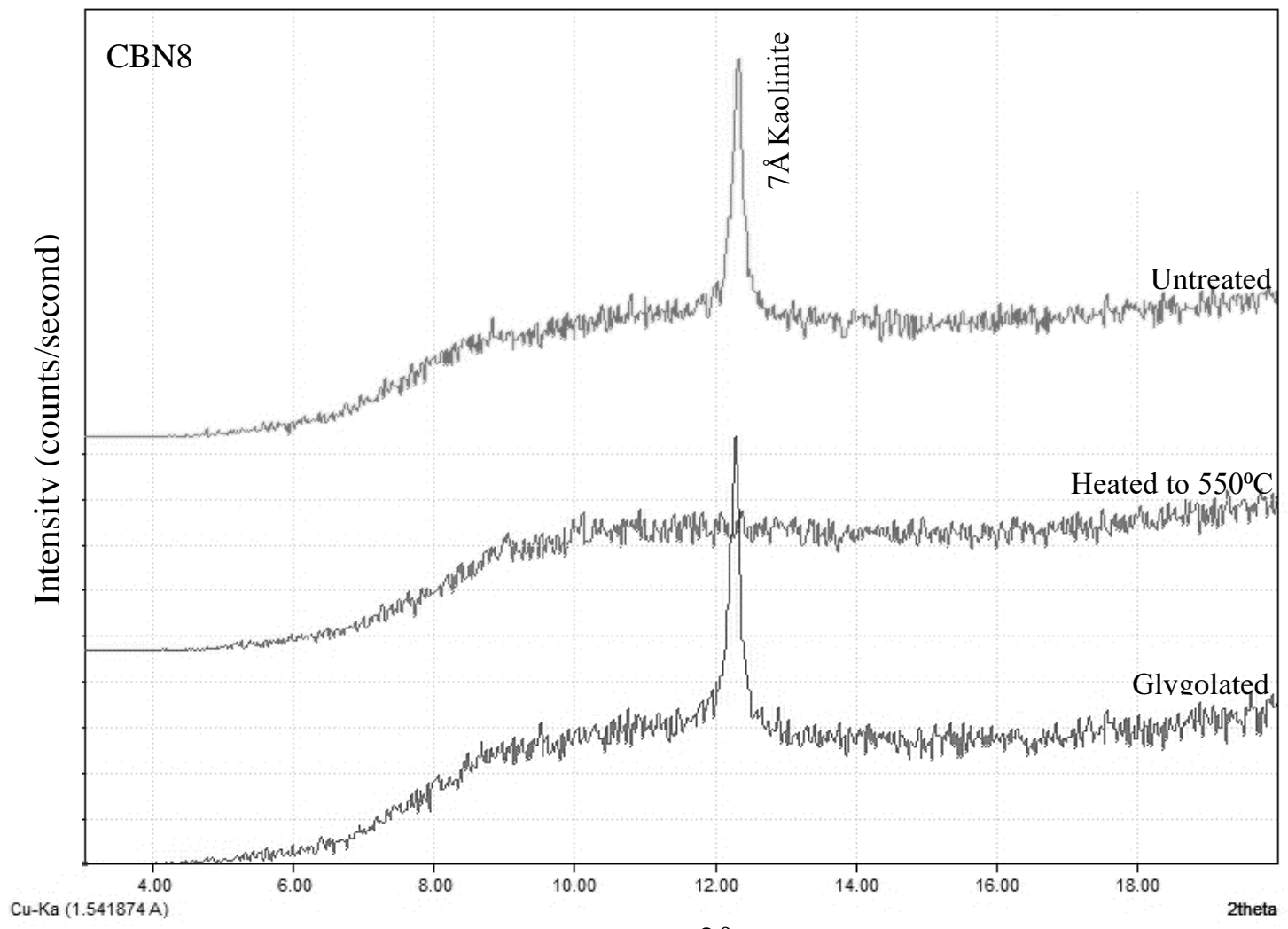

$2 \theta$

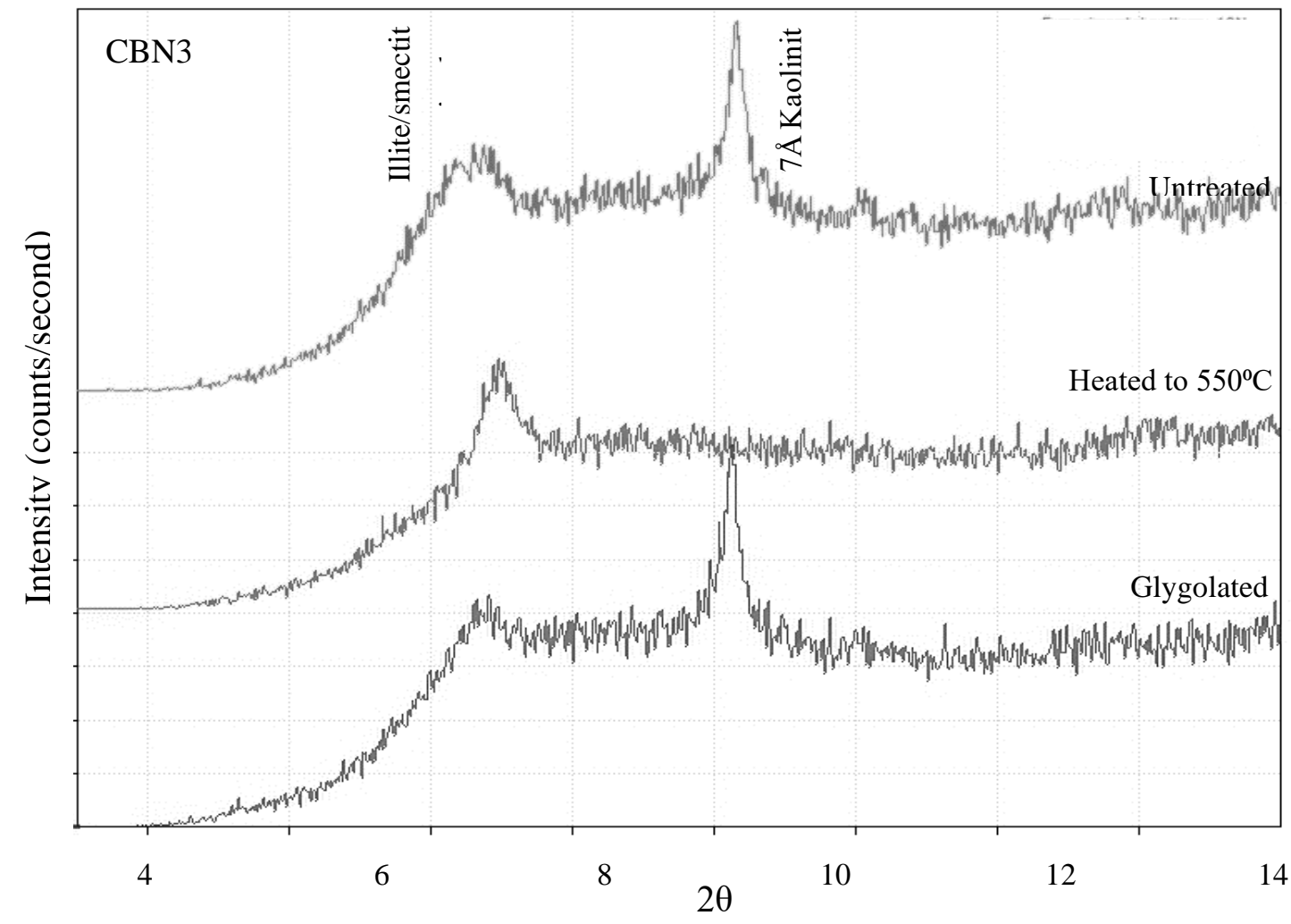

Fig. 4. X-ray diffractograms of selected shale samples from Barsarin and Banik sections, Chia Gara Formation (oriented) 


\subsection{Geochemistry}

\subsubsection{Major oxides}

Major oxides were analyzed in order to employ them for establish the mineral/ element association. The correlation coefficient indicates the general trends (Fu et al. 2010). Major oxide contents are listed in Table 1. The most abundant oxide is $\mathrm{SiO}_{2}$ range between $20.80-46.50 \%$ with an average $33.19 \%$ in the Chia Gara black shale. It followed by $\mathrm{CaO}(11.55-32.40 \%$, average $=20.54 \%), \mathrm{Al}_{2} \mathrm{O}_{3}(5.78-$ $23.30 \%$, average $=12.08 \%), \mathrm{Fe}_{2} \mathrm{O}_{3}(2.20-8.65 \%$; average $=4.73 \%)$ and $\mathrm{K}_{2} \mathrm{O}(0.52-3.97 \%$, average $=$ $1.71 \%$ ). However, $\mathrm{MgO}, \mathrm{Na}_{2} \mathrm{O}, \mathrm{TiO}_{2}, \mathrm{MnO}$ and $\mathrm{P}_{2} \mathrm{O}_{5}$ contents are less abundant, less than $1 \%$ (Table 1). The average content of $\mathrm{CaO}$ is higher than the geochemical standards (PAAS; Taylor and McLennan, 1985) indicating the prominent of carbonates in the environment of deposition for the Chia Gara shale that occurred in open marine setting as reported by many workers (i.e., Mohialdeen et al. 2013; Mustafa and Tobia, 2020). On the other hand, $\mathrm{SiO}_{2}, \mathrm{Al}_{2} \mathrm{O}_{3}, \mathrm{Fe}_{2} \mathrm{O}_{3}, \mathrm{~K}_{2} \mathrm{O}, \mathrm{MgO}, \mathrm{Na}_{2} \mathrm{O}, \mathrm{TiO}_{2}$ and $\mathrm{MnO}$ exhibited lower contents compared to that of PAAS, while $\mathrm{P}_{2} \mathrm{O}_{5}$ is nearly similar to PAAS (Table 1). The correlation coefficients among major elements are given in Table 2. CaO is negatively correlated with $\mathrm{SiO}_{2}, \mathrm{Al}_{2} \mathrm{O}_{3}, \mathrm{Fe}_{2} \mathrm{O}_{3}$ and $\mathrm{TiO}_{2}$. This suggests that these oxides are not associated to the carbonate phase. On the other hand, significant positive correlation between $\mathrm{Al}_{2} \mathrm{O}_{3}$ and $\mathrm{Fe}_{2} \mathrm{O}_{3}(\mathrm{r}=0.905)$ and $\mathrm{TiO}_{2}(\mathrm{r}=$ 0.943), indicating these elements are from terrestrial origin, and transported as detrital components.

Table 1. Clay parameters (in clay fraction), TOC and major oxides (in wt \%), and REE parameters (bulk sample) for the Chia Gara black shale

\begin{tabular}{|c|c|c|c|c|c|c|c|c|c|c|c|c|c|c|c|c|c|}
\hline Sam & CBN & CBN & CBN & CBN & CBN & CBN & CBN & CBN & CBN & CBK & CBK & CBK & CBK & CBK & CBK & Ave. & PAS \\
\hline Kaol & 63.64 & 8.54 & 34.78 & 24.62 & 86.15 & 62.46 & 100 & 100 & 84 & 58.82 & - & - & 69.57 & 100 & 95.39 & 59.2 & \\
\hline Illite & 36.36 & 91.46 & 65.22 & - & 13.85 & 37.54 & - & - & 16 & 41.18 & 100 & 100 & 30.43 & - & 4.61 & 35.78 & \\
\hline Kaol & 0.133 & 0.5 & 0.063 & 0.085 & 0.143 & 0.067 & 0.077 & 0.045 & 0.048 & 0.113 & - & - & 0.038 & 0.107 & 0.087 & 0.1 & \\
\hline Illite & 0.65 & 0.8 & 0.65 & - & 0.42 & 0.68 & - & - & 0.6 & 0.63 & 0.5 & 0.37 & 0.37 & - & 0.43 & 0.56 & \\
\hline TOC & 1.52 & 1.61 & 1.86 & 2.17 & 2.5 & 1.47 & 1.92 & 1.76 & 1.92 & 4.19 & 5.87 & 5.5 & 3.56 & 3.08 & 2.08 & 2.73 & \\
\hline $\mathrm{SiO}_{2}$ & 25.4 & 36 & 46.5 & 23.2 & 33.4 & 33.9 & 34.9 & 38.4 & 31.7 & 45.3 & 27.8 & 32.6 & 32.6 & 35.3 & 20.8 & 33.19 & 62.4 \\
\hline $\mathrm{Al}_{2} \mathrm{O}$ & 12.25 & 6.47 & 13.4 & 5.78 & 9.26 & 8.15 & 16.55 & 12.25 & 17.1 & 23.3 & 10.85 & 9.29 & 9.29 & 15.35 & 11.9 & 12.08 & 18.78 \\
\hline $\mathrm{Fe}_{2} \mathrm{O}$ & 6.48 & 2.9 & 6.39 & 2.2 & 3.52 & 2.94 & 6.54 & 4.03 & 7.12 & 8.65 & 3.45 & 3.72 & 3.72 & 6.66 & 2.69 & 4.73 & 7.18 \\
\hline $\mathrm{CaO}$ & 24.2 & 25.4 & 12.8 & 31.9 & 23.6 & 26.5 & 11.55 & 17.75 & 18.85 & 0.84 & 23.3 & 22.1 & 22.1 & 14.75 & 32.4 & 20.54 & 1.29 \\
\hline $\mathrm{MgO}$ & 0.81 & 0.49 & 0.72 & 1.84 & 0.66 & 0.41 & 0.53 & 0.27 & 0.44 & 1.48 & 0.89 & 0.65 & 0.65 & 0.65 & 0.58 & 0.74 & 2.19 \\
\hline $\mathrm{Na}_{2} \mathrm{O}$ & 0.04 & 0.58 & 0.35 & 0.02 & 0.05 & 0.04 & 0.08 & 0.02 & 0.1 & 0.05 & 0.05 & 0.52 & 0.52 & 0.01 & 0.03 & 0.16 & 1.19 \\
\hline $\mathrm{K}_{2} \mathrm{O}$ & 1.65 & 0.82 & 1.51 & 0.52 & 0.98 & 0.81 & 1.06 & 0.55 & 0.86 & 3.5 & 2.95 & 3.97 & 3.97 & 1.71 & 0.75 & 1.71 & 3.68 \\
\hline $\mathrm{MnO}$ & 0.02 & 0.01 & 0.02 & 0.01 & 0.01 & 0.01 & 0.01 & 0.01 & 0.01 & 0.03 & 0.01 & 0.01 & 0.01 & 0.01 & 0.01 & 0.01 & 0.11 \\
\hline $\mathrm{TiO}_{2}$ & 0.66 & 0.4 & 0.85 & 0.36 & 0.59 & 0.56 & 1.05 & 0.71 & 0.92 & 1.13 & 0.48 & 0.51 & 0.51 & 0.93 & 0.76 & 0.69 & 0.99 \\
\hline $\mathrm{P}_{2} \mathrm{O}_{5}$ & 0.54 & 0.03 & 0.08 & 0.01 & 0.1 & 0.05 & 0.14 & 0.13 & 0.21 & 0.2 & 0.28 & 0.22 & 0.22 & 0.4 & 0.08 & 0.18 & 0.16 \\
\hline LOI & 26.3 & 25 & 17.75 & 30 & 22.4 & 22.9 & 20.1 & 19.7 & 22.8 & 16.95 & 28.6 & 25.5 & 28.3 & 22.6 & 30.7 & 23.97 & 6 \\
\hline Total & 98.35 & 98.1 & 100.3 & 95.84 & 94.57 & 96.27 & 92.51 & 93.82 & 100.1 & 101.4 & 98.66 & 99.09 & 99.92 & 98.37 & 100.7 & 97.87 & \\
\hline REE & 118.7 & 101.9 & 194.4 & 92.52 & 113.3 & 116.0 & 179.2 & 157.2 & 185.3 & 177.1 & 103.6 & 109.7 & 114. & 182.5 & 127.7 & 138.2 & \\
\hline $\mathrm{Ce} / \mathrm{C}$ & 1.07 & 0.96 & 1.01 & 1.01 & 0.95 & 0.97 & 0.98 & 0.99 & 0.98 & 1.03 & 0.96 & 0.87 & 0.89 & 0.97 & 0.85 & 0.97 & \\
\hline $\mathrm{Eu} / \mathrm{E}$ & 0.69 & 0.74 & 0.66 & 0.72 & 0.59 & 0.7 & 0.66 & 0.64 & 0.68 & 0.64 & 0.73 & 0.63 & 0.68 & 0.65 & 0.7 & 0.67 & \\
\hline
\end{tabular}


Table 2A. Correlation coefficients matrix for clay minerals, major and trace elements in the black shale of Chia Gara Formation

\begin{tabular}{|c|c|c|c|c|c|c|c|c|c|c|c|c|c|c|}
\hline & Kaol & Illite & TOC & $\mathrm{SiO}_{2}$ & $\mathbf{A l}_{2} \mathbf{O}_{3}$ & $\mathrm{Fe}_{2} \mathrm{O}_{3}$ & $\mathrm{CaO}$ & MgO & $\mathrm{Na}_{2} \mathrm{O}$ & $\mathbf{K}_{2} \mathbf{O}$ & MnO & $\mathrm{Tio}_{2}$ & $\mathbf{P}_{2} \mathbf{O}_{5}$ & LOI \\
\hline Kaol & 1 & & & & & & & & & & & & & \\
\hline Illite & -.862 & 1 & & & & & & & & & & & & \\
\hline TOC & -.489 & .546 & 1 & & & & & & & & & & & \\
\hline $\mathrm{SiO}_{2}$ & .021 & .182 & .011 & 1 & & & & & & & & & & \\
\hline $\mathbf{A l}_{2} \mathbf{O}$ & .432 & -.232 & .108 & .471 & 1 & & & & & & & & & \\
\hline $\mathrm{Fe}_{2} \mathrm{O}$ & .328 & -.145 & -.005 & .537 & .905 & 1 & & & & & & & & \\
\hline $\mathrm{CaO}$ & -.211 & .009 & -.187 & -.818 & -.844 & -.851 & 1 & & & & & & & \\
\hline Mg & -.363 & -.027 & .267 & -.123 & .068 & .063 & -.056 & 1 & & & & & & \\
\hline $\mathrm{Na}_{2}$ & -.523 & .621 & .198 & .251 & -.347 & -.201 & .042 & -.206 & 1 & & & & & \\
\hline $\mathrm{K}_{2} \mathrm{O}$ & -.379 & .516 & .831 & .198 & .206 & .202 & -.347 & .203 & .441 & 1 & & & & \\
\hline Mn & -.067 & .133 & .069 & .502 & .627 & .672 & -.639 & .450 & -.095 & .320 & 1 & & & \\
\hline $\mathrm{TiO}_{2}$ & .596 & -.391 & -.111 & .480 & .943 & .864 & -.793 & -.077 & -.370 & .001 & .501 & 1 & & \\
\hline $\mathbf{P}_{2} \mathrm{O}_{5}$ & .127 & .044 & .271 & -.138 & .340 & .500 & -.217 & -.038 & -.171 & .397 & .252 & .226 & 1 & \\
\hline LOI & -.285 & .081 & .177 & -.899 & -.627 & -.678 & .844 & .175 & .087 & .047 & -.502 & -.682 & .053 & 1 \\
\hline $\mathbf{R b}$ & -.050 & .214 & .551 & .302 & .738 & .680 & -.664 & .365 & -.140 & .670 & .768 & .524 & .551 & -.289 \\
\hline $\mathrm{Sr}$ & .008 & .154 & -.451 & .369 & -.294 & -.246 & .094 & -.521 & .243 & -.427 & -.202 & -.101 & -.588 & -.333 \\
\hline Ba & -.110 & .307 & .678 & .394 & .725 & .640 & -.694 & .257 & -.005 & .729 & .652 & .525 & .481 & -.326 \\
\hline Th & .527 & -.348 & -.099 & .552 & .944 & .886 & -.849 & -.061 & -.348 & .000 & .499 & .968 & .196 & -.754 \\
\hline $\mathbf{U}$ & -.299 & .371 & .322 & -.262 & .011 & .175 & .040 & .200 & -.020 & .416 & .363 & -.173 & .798 & .263 \\
\hline $\mathbf{Y}$ & .207 & -.060 & .078 & .437 & .390 & .441 & -.442 & -.203 & .217 & .173 & .079 & .531 & .091 & -.372 \\
\hline $\mathbf{Z r}$ & .701 & -.523 & -.222 & .396 & .844 & .758 & -.690 & -.178 & -.387 & -.127 & .315 & .958 & .115 & -.652 \\
\hline Nb & .657 & -.501 & -.332 & .455 & .821 & .775 & -.699 & -.170 & -.389 & -.224 & .364 & .928 & .063 & -.730 \\
\hline Hf & .689 & -.501 & -.244 & .480 & .838 & .741 & -.708 & -.196 & -.364 & -.144 & .359 & .944 & .038 & -.717 \\
\hline Sc & .296 & -.079 & .192 & .527 & .927 & .869 & -.844 & .144 & -.282 & .316 & .755 & .873 & .407 & -.608 \\
\hline $\mathbf{V}$ & -.018 & .199 & .574 & .190 & .425 & .499 & -.454 & -.061 & .026 & .565 & .114 & .385 & .634 & -.202 \\
\hline $\mathrm{Cr}$ & .407 & -.232 & .097 & -.068 & .479 & .303 & -.152 & -.219 & -.085 & .091 & .018 & .560 & .141 & .007 \\
\hline Co & -.029 & .129 & .029 & .093 & .436 & .594 & -.365 & .276 & -.134 & .285 & .755 & .297 & .705 & -.178 \\
\hline $\mathbf{N i}$ & -.157 & .336 & .522 & .168 & .130 & .297 & -.291 & -.090 & .364 & .658 & .112 & .090 & .667 & -.044 \\
\hline $\mathrm{Cu}$ & -.304 & .472 & .595 & -.031 & .244 & .364 & -.241 & .110 & .140 & .715 & .388 & .047 & .837 & .077 \\
\hline Zn & -.024 & .291 & .089 & .445 & .508 & .756 & -.604 & -.223 & .159 & .333 & .415 & .485 & .601 & -.495 \\
\hline Mo & -.252 & .376 & .363 & -.044 & .119 & .304 & -.166 & .078 & -.029 & .408 & .331 & -.037 & .851 & .029 \\
\hline REE & .518 & -.333 & -.197 & .619 & .819 & .822 & -.778 & -.192 & -.221 & -.087 & .379 & .910 & .140 & -.755 \\
\hline
\end{tabular}

\subsubsection{Trace elements}

Their concentrations in the shale of Chia Gara Formation are listed in Table 3. The V and $\mathrm{Sr}$ record the highest concentrations with an average 582 and $542 \mathrm{ppm}$, respectively. $\mathrm{Zr}, \mathrm{Cr}, \mathrm{Ni}, \mathrm{Zn}$ and $\mathrm{Mo}$ relatively have high concentrations (less than $100 \mathrm{ppm}$ in average). $\mathrm{Ba}$ and $\mathrm{Cu}$ concentrations are less than $100 \mathrm{ppm}$ and $\mathrm{Rb}, \mathrm{Th}, \mathrm{U}, \mathrm{Y}, \mathrm{Nb}, \mathrm{Hf}, \mathrm{Sc}$, and Co, exist in low concentrations having averages of $<40$ ppm. The correlation coefficients ( $\mathrm{r}$ ) between major and trace elements were calculated. $\mathrm{Al}_{2} \mathrm{O}_{3}$ and $\mathrm{TiO}_{2}$ represent significant positive correlations with $\mathrm{Th}, \mathrm{Sc}, \mathrm{Hf}, \mathrm{Zr}, \mathrm{Nb}, \mathrm{Rb}$ and $\mathrm{Ba}$. $\mathrm{Fe}_{2} \mathrm{O}_{3}$ has significant positive correlations with Th. Sc, Nb, Zr, Zn, Hf, Rb, Ba and Co. Ba, Rb, Zr, Th, Hf, Nb, Sc, Zn and Co. $\mathrm{CaO}$ have significant -ve correlations with most trace elements. $\mathrm{K}_{2} \mathrm{O}$ shows significant positive correlations with $\mathrm{Ba}, \mathrm{Cu}, \mathrm{Rb}, \mathrm{Ni}$ and $\mathrm{V} . \mathrm{P}_{2} \mathrm{O}_{5}$ reveals significant positive correlations with $\mathrm{Mo}, \mathrm{Cu}, \mathrm{U}$, $\mathrm{Co}, \mathrm{Ni}, \mathrm{V}, \mathrm{Zn}$ and $\mathrm{Rb}$ (Table 2).

\subsubsection{Total organic matter}

The contents of total organic carbon (TOC) of the studied black shale are ranged between 1.47 to $5.87 \mathrm{wt} \%$ with an average $2.73 \mathrm{wt} \%$ (Table 1). There is decreasing tendency in the distribution of TOC for the studied shales in Banik section. TOC contents represent positive correlations with $\mathrm{K}_{2} \mathrm{O}$ (reflect the illite content) and $\mathrm{Ba}$ (Table 2). 
Table 2B. Correlation coefficients for major and trace elements of the Chia Gara Formation, Correlation is significant at the 0.01 level (2-tailed); Correlation is significant at the 0.05 level (2-tailed); no. of samples $=15$

\begin{tabular}{|c|c|c|c|c|c|c|c|c|c|c|c|c|c|c|c|c|c|c|}
\hline & $\mathbf{R b}$ & $\mathrm{Sr}$ & $\mathbf{B a}$ & Th & $\mathbf{U}$ & $\mathbf{Y}$ & $\mathbf{Z r}$ & Nb & Hf & Sc & $\mathbf{V}$ & $\mathrm{Cr}$ & Co & $\mathrm{Ni}$ & $\mathrm{Cu}$ & $\mathrm{Zn}$ & Mo & REE \\
\hline $\mathbf{R b}$ & 1 & & & & & & & & & & & & & & & & & \\
\hline $\mathrm{Sr}$ & -.521 & 1 & & & & & & & & & & & & & & & & \\
\hline $\mathbf{B a}$ & .939 & -.475 & 1 & & & & & & & & & & & & & & & \\
\hline Th & .505 & -.119 & .508 & 1 & & & & & & & & & & & & & & \\
\hline $\mathbf{U}$ & .502 & -.506 & .361 & -.186 & 1 & & & & & & & & & & & & & \\
\hline $\mathbf{Y}$ & .106 & .108 & .324 & .449 & -.313 & 1 & & & & & & & & & & & & \\
\hline $\mathbf{Z r}$ & .320 & -.029 & .325 & .938 & -.309 & .506 & 1 & & & & & & & & & & & \\
\hline $\mathbf{N b}$ & .270 & .040 & .249 & .947 & -.308 & .402 & .975 & 1 & & & & & & & & & & \\
\hline Hf & .302 & .043 & .321 & .939 & -.355 & .496 & .983 & .976 & 1 & & & & & & & & & \\
\hline Sc & .815 & -.254 & .800 & .832 & .173 & .460 & .720 & .677 & .717 & 1 & & & & & & & & \\
\hline $\mathbf{V}$ & .516 & -.351 & .649 & .342 & .286 & .588 & .303 & .184 & .218 & .483 & 1 & & & & & & & \\
\hline $\mathrm{Cr}$ & .215 & -.163 & .372 & .415 & -.216 & .682 & .546 & .399 & .519 & .444 & .410 & 1 & & & & & & \\
\hline Co & .696 & -.432 & .506 & .279 & .796 & $\overline{-} \cdot 151$ & .132 & .170 & .097 & .548 & .249 & -.089 & 1 & & & & & \\
\hline $\mathbf{N i}$ & .395 & -.330 & .520 & .032 & .482 & .520 & -.002 & -.116 & -.068 & .296 & .840 & .242 & .344 & 1 & & & & \\
\hline $\mathrm{Cu}$ & .692 & -.573 & .672 & .036 & .855 & .022 & -.104 & -.157 & -.163 & .369 & .670 & .051 & .716 & .761 & 1 & & & \\
\hline $\mathbf{Z n}$ & .463 & -.023 & .442 & .527 & .392 & .391 & .409 & .438 & .369 & .551 & .602 & .067 & .543 & .532 & .566 & 1 & & \\
\hline Mo & .508 & -.471 & .444 & -.026 & .923 & $\begin{array}{c}- \\
.118\end{array}$ & -.176 & -.179 & -.226 & .282 & .500 & -.188 & .754 & .652 & .887 & .527 & 1 & \\
\hline REE & .309 & .047 & .385 & .925 & -.307 & .683 & .883 & .885 & .902 & .752 & .357 & .494 & .151 & .106 & -.078 & .537 & -.104 & 1 \\
\hline
\end{tabular}

\section{Discussion}

\subsection{Enrichment of the Trace Elements}

Controls on trace elements behavior in black shale can be deduced from the enrichment factors. Table 3 represents the abundance of the trace elements in the studied black shale. $\mathrm{Ba}, \mathrm{Rb}, \mathrm{Y}, \mathrm{Th}, \mathrm{Hf}, \mathrm{Zr}$, $\mathrm{Co}$ and $\mathrm{Sc}$ are less than the averages reported for PAAS, while $\mathrm{Y}$ and $\mathrm{Nb}$ have been found to be similar averages. In contrast, $\mathrm{Sr}, \mathrm{U}, \mathrm{V}, \mathrm{Cr}, \mathrm{Ni}, \mathrm{Cu}, \mathrm{Zn}$ and Mo concentrations are much higher than the PAAS. By contrast, $\mathrm{Rb}, \mathrm{Ba}, \mathrm{V}, \mathrm{Ni}$ and $\mathrm{Cu}$ are associated with organic matter (represented as TOC) as confirmed by their significant correlation coefficients (Table 2). The negative correlation of $\mathrm{Sr}$ with TOC could be assigning to the adsorption of $\mathrm{Sr}$ on clay minerals and/or may enter the calcium lattice defects (Aziz et al. 2008) or the black shale was subjected to severe diagenetic processes.

The enrichment of the elements in this study were estimated by using the enrichment factor (EF) described by Brumsack (2006) as follows:

$$
\mathrm{EF}=(\mathrm{X} / \mathrm{Al})_{\text {sample }} /(\mathrm{X} / \mathrm{Al}) \text { PAAS }
$$

Where $\mathrm{X}$ and $\mathrm{Al}$ are the element and aluminum concentrations, respectively. The elements are normalized to $\mathrm{Al}$ for mineralogical variability and grain size compensation, since it represents aluminosilicates (Luoma and Rainbow, 2008). The calculated EFs are presented in Table 3. The studied trace elements were classified to four categories based on the enrichment levels for Sutherland (2000), weakly enriched (Th, Y, Zr, Nb, Hf, Cr, Co, Cu); moderately enriched ( $\mathrm{Sr}, \mathrm{Zn}$ ); significantly enriched $(\mathrm{U}, \mathrm{V}, \mathrm{Ni})$; and extremely enriched (Mo). There is depletion in $\mathrm{Rb}, \mathrm{Ba}$, and Sc. The EFs were in the order $\mathrm{Ba}<\mathrm{Rb}<\mathrm{Sc}<\mathrm{Th}<\mathrm{Zr}<\mathrm{Hf}<\mathrm{Co}<\mathrm{Y}<\mathrm{Nb}<\mathrm{Cr}<\mathrm{Cu}<\mathrm{Zn}<\mathrm{Sr}<\mathrm{Ni}<\mathrm{V}<\mathrm{U}<\mathrm{Mo}$. The highest enrichment was recorded for Mo because of its association with phosphate as $\mathrm{P}_{2} \mathrm{O}_{5}$ (Silva and Bustin, 2020) and with organic matter (Spear and Zheng, 1999; Tribovillard et al. 2004; Fu et al. 2011). 
Table 3. Trace elements concentrations (in ppm) and enrichment factors in the black shale form the Chia Gara Formation with averaged values in PAAS

\begin{tabular}{|c|c|c|c|c|c|c|c|c|c|c|c|c|c|c|c|c|c|}
\hline Sample & $\mathbf{R b}$ & $\mathrm{Sr}$ & $\mathbf{B a}$ & Th & $\mathbf{U}$ & $\mathbf{Y}$ & $\mathbf{Z r}$ & $\mathrm{Nb}$ & Hf & Sc & $\mathbf{V}$ & $\mathrm{Cr}$ & Co & $\mathrm{Ni}$ & $\mathrm{Cu}$ & $\mathbf{Z n}$ & Mo \\
\hline CBN1 & 57.5 & 108 & 91.9 & 8.86 & 51.4 & 15.7 & 132 & 18 & 3 & 11 & 619 & 90 & 65 & 283 & 141 & 184 & 502 \\
\hline $\mathrm{CBN} 2$ & 16.4 & 747 & 54.9 & 5.36 & 15 & 18.2 & 81 & 10.6 & 1.8 & 6 & 287 & 70 & 16 & 166 & 41 & 116 & 155 \\
\hline CBN3 & 34.2 & 817 & 94.1 & 12.1 & 10.85 & 32.1 & 169 & 23.4 & 4.6 & 13 & 564 & 150 & 15 & 224 & 40 & 177 & 128 \\
\hline CBN4 & 13.2 & 116 & 36 & 5.26 & 11.3 & 16.4 & 92 & 11.6 & 2 & 5 & 287 & 60 & 6 & 81 & 17 & 63 & 63 \\
\hline CBN5 & 26.4 & 725 & 63.8 & 7.54 & 14.4 & 18.8 & 139 & 17.9 & 3.6 & 8 & 440 & 90 & 8 & 157 & 30 & 99 & 120 \\
\hline CBN6 & 20.8 & 868 & 47.0 & 7.39 & 7.23 & 17.6 & 125 & 16.4 & 3 & 7 & 482 & 80 & 7 & 100 & 30 & 122 & 56 \\
\hline CBN7 & 34.4 & 465 & 73.3 & 15.25 & 7.76 & 23.9 & 235 & 31.8 & 5.6 & 12 & 677 & 140 & 14 & 189 & 40 & 168 & 111 \\
\hline CBN8 & 20.7 & 378 & 69.0 & 11.4 & 10.3 & 18.5 & 161 & 22.6 & 4.4 & 9 & 368 & 100 & 8 & 139 & 36 & 100 & 172 \\
\hline CBN9 & 36.3 & 363 & 91.1 & 14.15 & 7.98 & 23.5 & 195 & 27.6 & 5 & 10 & 604 & 190 & 10 & 108 & 43 & 160 & 58 \\
\hline CBK1 & 105.5 & 132 & 198 & 16.3 & 20.5 & 21.4 & 200 & 27 & 5.2 & 18 & 686 & 150 & 46 & 197 & 95 & 144 & 206 \\
\hline CBK2 & 58.4 & 232 & 126 & 7.04 & 31.9 & 16.3 & 91 & 9.8 & 2 & 10 & 697 & 90 & 15 & 212 & 102 & 135 & 333 \\
\hline CBK3 & 44.1 & 268 & 127.5 & 6.97 & 22.2 & 26 & 117 & 13 & 2.7 & 8 & 1020 & 170 & 13 & 400 & 124 & 147 & 266 \\
\hline CBK4 & 40.6 & 268 & 88.7 & 6.79 & 17.8 & 22.2 & 114 & 12.3 & 2.7 & 8 & 522 & 120 & 11 & 269 & 71 & 126 & 121 \\
\hline CBK5 & 47.2 & 227 & 121 & 11.95 & 17.05 & 32.6 & 177 & 20.7 & 4.1 & 13 & 1100 & 210 & 19 & 393 & 84 & 150 & 278 \\
\hline CBK6 & 30.4 & 392 & 79.9 & 8.4 & 10.45 & 23.5 & 162 & 18.5 & 4 & 10 & 381 & 280 & 7 & 118 & 31 & 68 & 23 \\
\hline Average & 39.07 & 542.1 & 90.81 & 9.65 & 17.07 & 21.78 & 146 & 18.75 & 3.58 & 9.87 & 582.3 & 132.7 & 17.33 & 202.4 & 61.67 & 130.6 & 172.8 \\
\hline PASS & 160 & 200 & 650 & 14.6 & 3.1 & 27 & 210 & 19 & 5 & 16 & 150 & 110 & 23 & 55 & 50 & 85 & 1.0 \\
\hline \multicolumn{18}{|c|}{ Enrichment factors (EF) } \\
\hline CBN1 & 0.55 & 0.84 & 0.22 & 0.94 & 25.58 & 0.90 & 0.97 & 1.46 & 0.93 & 1.06 & 6.37 & 1.26 & 4.36 & 7.94 & 4.35 & 3.34 & 775 \\
\hline CBN2 & 0.30 & 10.91 & 0.25 & 1.07 & 14.13 & 1.97 & 1.13 & 1.63 & 1.05 & 1.10 & 5.59 & 1.86 & 2.03 & 8.82 & 2.40 & 3.99 & 453 \\
\hline CBN3 & 0.30 & 5.76 & 0.20 & 1.17 & 4.94 & 1.68 & 1.14 & 1.74 & 1.30 & 1.15 & 5.30 & 1.92 & 0.92 & 5.74 & 1.13 & 2.94 & 181 \\
\hline CBN4 & 0.27 & 1.90 & 0.18 & 1.18 & 11.92 & 1.99 & 1.43 & 2.00 & 1.31 & 1.02 & 6.26 & 1.78 & 0.85 & 4.82 & 1.11 & 2.42 & 206 \\
\hline CBN5 & 0.34 & 7.40 & 0.20 & 1.05 & 9.48 & 1.42 & 1.35 & 1.92 & 1.47 & 1.02 & 5.99 & 1.67 & 0.71 & 5.83 & 1.22 & 2.38 & 245 \\
\hline CBN6 & 0.30 & 10.06 & 0.17 & 1.17 & 5.41 & 1.51 & 1.38 & 2.00 & 1.39 & 1.01 & 7.45 & 1.69 & 0.71 & 4.22 & 1.39 & 3.33 & 130 \\
\hline CBN7 & 0.25 & 2.66 & 0.13 & 1.19 & 2.86 & 1.01 & 1.28 & 1.91 & 1.28 & 0.86 & 5.15 & 1.45 & 0.70 & 3.92 & 0.91 & 2.26 & 127 \\
\hline CBN8 & 0.20 & 2.92 & 0.16 & 1.20 & 5.13 & 1.06 & 1.18 & 1.84 & 1.36 & 0.87 & 3.79 & 1.40 & 0.54 & 3.90 & 1.11 & 1.82 & 265 \\
\hline CBN9 & 0.25 & 2.01 & 0.15 & 1.07 & 2.85 & 0.96 & 1.03 & 1.61 & 1.11 & 0.69 & 4.45 & 1.91 & 0.48 & 2.17 & 0.95 & 2.08 & 64 \\
\hline CBK1 & 0.53 & 0.54 & 0.25 & 0.91 & 5.36 & 0.64 & 0.77 & 1.15 & 0.84 & 0.91 & 3.71 & 1.11 & 1.62 & 2.91 & 1.54 & 1.37 & 167 \\
\hline CBK2 & 0.64 & 2.02 & 0.34 & 0.84 & 17.93 & 1.05 & 0.75 & 0.90 & 0.70 & 1.09 & 8.09 & 1.43 & 1.14 & 6.71 & 3.55 & 2.77 & 580 \\
\hline CBK3 & 0.56 & 2.73 & 0.40 & 0.97 & 14.57 & 1.96 & 1.13 & 1.39 & 1.10 & 1.02 & 13.83 & 3.14 & 1.15 & 14.80 & 5.05 & 3.52 & 541 \\
\hline CBK4 & 0.52 & 2.73 & 0.28 & 0.95 & 11.68 & 1.67 & 1.10 & 1.32 & 1.10 & 1.02 & 7.08 & 2.22 & 0.97 & 9.95 & 2.89 & 3.02 & 246 \\
\hline CBK5 & 0.36 & 1.40 & 0.23 & 1.01 & 6.77 & 1.49 & 1.04 & 1.34 & 1.01 & 1.00 & 9.03 & 2.35 & 1.02 & 8.80 & 2.07 & 2.17 & 342 \\
\hline CBK6 & 0.30 & 3.11 & 0.20 & 0.91 & 5.35 & 1.38 & 1.23 & 1.55 & 1.27 & 0.99 & 4.03 & 4.04 & 0.48 & 3.41 & 0.98 & 1.27 & 37 \\
\hline Average & 0.38 & 3.18 & 0.22 & 1.03 & 8.62 & 1.26 & 1.09 & 1.54 & 1.12 & 0.96 & 6.07 & 1.89 & 1.18 & 5.76 & 1.93 & 2.40 & 270 \\
\hline
\end{tabular}

The positive correlation between Mo and other redox sensitive trace elements $(\mathrm{U}, \mathrm{Co}, \mathrm{Ni}$ and $\mathrm{Cu}$ ) as shown in Table 2; and the anoxic environment of deposition was confirmed by $\mathrm{U} / \mathrm{Th}=1.77$ that is more than 1.25 (Jones and Manning, 1994). Also the Mo is associated with clay minerals (especially illite). Therefore, the extreme enrichment with Mo (EF= 270.37) and significantly enrichment in U, V and $\mathrm{Ni}(\mathrm{EF}=8.62,6.07$ and 5.76, respectively) in the studied samples suggested that the black shale of Chia Gara Formation was related to anoxic bottom waters (Algeo and Maynard, 2004; Fu et al. 2017). 


\subsection{Minerals and Trace Elements Associations}

The significant positive correlation between $\mathrm{K}_{2} \mathrm{O}$ (as illite) and TOC in the studied black shale could be attributed to the high amphoteric properties in the broken edges in the illite tend to adsorb the organic matter (Wiseman and Püttmann, 2006). The negative correlation with the kaolinite mineral could be related to its little surface area and weak exchange properties. There is trace of Namontmorillonite as mixed layer with illite that was difficult to detect from X-ray diffractograms, this denoted by the positive relationship between illite and $\mathrm{K}_{2} \mathrm{O}$ with $\mathrm{Na}_{2} \mathrm{O}$ (Table 2).

The elements $\mathrm{Ti}, \mathrm{Zr}$, Th, Hf, Nb, and REE are connected with clastic phase (especially kaolinite mineral) and $\mathrm{Rb}, \mathrm{Ba}, \mathrm{V}, \mathrm{Ni}$, and $\mathrm{Cu}$ are accompanied with organic matter and lesser extend with illite (Table 2). No association of any elements to carbonate phase, this confirmed from the negative correlation between $\mathrm{CaO}$ and other elements (Table 2). The iron minerals are adsorbed or coated the kaolinite particles (Liang and Morgan, 1990). In the studied black shale, uranium is essentially concentrated in the lattice of the apatite replacing Ca sites (Rakovan et al. 2002), and also adsorbed on the surface of the crystallites (Lucas and Abbas, 1989), this is confirmed by significant positive correlation between $\mathrm{U}$ and $\mathrm{P}_{2} \mathrm{O}_{5}$ (Table 2). In addition, there is lesser association between uranium and organic matter (weak positive correlation of $U$ with TOC; Table 2). The REEs in black shale are closely associated with terrigenous clastic rocks, whose provides relatively stable materials from the land (Qing et al. 2014).

\subsection{Relationship between Diagenesis and Maturation}

The Tithonian-Berriasian black shale is an important hydrocarbon source rock in Northern Iraq. Thermal history data give a clear understanding of the process's conduction to the maturation of the OM and then generation of oil and natural gas from the Chia Gara shale. Cardott and Lambert (1985) enumerate several methods employed to estimate the maturation of the source rocks and temperatures corresponding to the generation of oil and natural gas $\left(100-200^{\circ} \mathrm{C}\right)$; among these methods: 1$)$ organic geochemistry; 2) vitrinite reflectance $\left(\mathrm{R}_{\mathrm{o}}\right)$; 3 ) clay mineralogy (I/S mixed layer and illite).

The increasing in the diagenetic/metamorphic degree cause the increase in illite crystallinity (IC values decreased). The transformation of the smectite to illite and IC were utilized by several researchers to partition the diagenetic/low-metamorphism zone (Rainer et al. 2002). The transformation includes 3 stages: early stage described by exist of individual smectite; middle stage characterized by the disappearance of smectite and forming of I/S mixed layer; late stage the I/S layer peak has been combined with peak of illite leading to the diagenetic illite formation. The illite of late diagenesis has IC more than $0.42^{\circ} \Delta 2 \theta$, whereas the anchizone is described by the IC values ranged between 0.25 and $0.42^{\circ} \Delta 2 \theta$ (Kubler and Jaboyedoff, 2000). According to Dellisanti et al. (2010) conclude that the overmature rocks are described by a long-range ordered illite/smectite with an illite content more than $85 \%$ and the IC within the range $0.45-0.65\left({ }^{\circ} \Delta 2 \theta\right)$. Based on the illite content $(>90 \%)$ and IC values $(0.37-$ $\left.0.80^{\circ} \Delta 2 \theta\right)$, most of the studied black shale samples of Chia Gara Formation are within the late diagenetic stage up to the limit of anchizone.

Clay minerals, particularly diagenetic illite are employed in estimating the degree of thermal maturation (Pevear, 1999). Like vitrinite reflectance $\left(R_{0}\right)$ is increase with the thermal maturity increases, the illite percentage in the illite/smectite mixed stratifications would be increased. The illite layer proportion in I/S mixed layer and the IC (Kubler index) are further temperature assignment used to conclude the temperature of OC maturation in oil sources (Pevear, 1999; Whittington, 2009).The Chia Gara shales are described by high contents of OC (1.47-5.87\%), this is because of conservation under anoxic conditions and high sedimentation rate that cause faster burial of the OC. Mohialdeen et al. (2013) conclude the sediments of the Chia Gara have a high oil, but low potential of gas generation derived from the high content of hydrocarbon-rich Type II and mixed Type II-III kerogen. They estimate 
the $\mathrm{T}_{\max }$ (temperature at maximum of $\mathrm{S} 2$ peak) between 426 and $442^{\circ} \mathrm{C}$, with vitrinite reflectance values from 0.50 to $0.80 \% \mathrm{R}_{\mathrm{o}}$ implying the Chia Gara rocks are mature and go into the early mature to peak mature oil window. In current study the illite percentage in I/S mixed layer is $>90 \%$ and the Kubler index (KI) ranges from 0.37 to $0.80\left({ }^{\circ} \Delta 2 \theta\right)$ as shown in Table 1 . The rocks with over-mature kerogen showed $\mathrm{T}_{\max }$ values from 435 to $465^{\circ} \mathrm{C}$ corresponding to illite $>85 \%$ (in I/S mixed layers) and Kubler index values between 0.45 and $0.65\left({ }^{\circ} \Delta 2 \theta\right)$. These rocks are related to the deep diagenetic zone up to the limit of the anchizone and to palaeotemperature between $150-200^{\circ} \mathrm{C}$ (Arkai et al. 2002). Meanwhile, the shales of Chia Gara Formation are related to deep diagenetic zone with a palaeotemperature ranged from 150 to $200^{\circ} \mathrm{C}$.

\section{Conclusions}

Based on mineralogical and geochemical examination of the Tithonian-Berriasian black shales from the Chia Gara Formation, Kurdistan Region, Iraq, suggest the following conclusions:

- Mineralogically, the black shales comprise mainly of non-clay (calcite) and clay (kaolinite and illite) minerals.

- The black shale samples are characterized by high TOC contents (1.47- 5.87\%).

- In comparison with PAAS, the black shales have high $\mathrm{CaO}, \mathrm{V}, \mathrm{U}, \mathrm{Sr}, \mathrm{Mo}, \mathrm{Ni}$ and $\mathrm{Zn}$, and low content of $\mathrm{Na}_{2} \mathrm{O}, \mathrm{MnO}, \mathrm{Ba}$ and $\mathrm{Rb}$, and have nearly similar $\mathrm{P}_{2} \mathrm{O}_{5}, \mathrm{Nb}$ and $\mathrm{Hf}$.

- Zn, U, V, Ni and Mo have high EF and are related to the phosphate minerals, in addition, the organic matters play a significant role in the enrichment of $\mathrm{V}$ and $\mathrm{Ni}$.

- The elements Fe, Mn, Ti, Ba, Rb, Zr, Th, Sc, Nb, Hf and REE are mainly associated with clay minerals.

- The high illite content and low IC values imply that most of the Chia Gara black shales are overmatured with little samples inside the anchimetamorphic zone.

- The relationship between $T_{\max }$, illite in $\mathrm{I} / \mathrm{S}$ and/or the Kubler Index could be used in prediction of palaeotemperature, and the estimated temperature for the studied black shale is in the range of 150$200^{\circ} \mathrm{C}$.

\section{Acknowledgements}

We are grateful to Dr. Hikmat S. Al-Jaleel for helping in the field work. The authors are very grateful to the reviewers, Editor in Chief Prof. Dr. Salih M. Awadh, the Secretary of Journal Mr. Samir R. Hijab, and the Technical Editors for their great efforts and valuable comments.

\section{References}

Al-Beyati, F.M., 1998. Organic Geochemical and Environmental Evaluation Study of Chia Gara Formation from Selected Boreholes in middle Iraq, Ph. D. Thesis, University of Baghdad, 172 pp.

Algeo, T.J., Maynard, J.B., 2004. Trace element behavior and redox facies in core shales of Upper Pennsylvanian Kansas-type cyclothems. Chemical Geology, 206, 289-318.

Al-Sharhan, A.S., Nairn, A.E.M., 1997. Sedimentary Basin and Petroleum Geology of the Middle East. Elsevier Science, 942.

Arkai, P., Sassi, F.P., Desmons, J., 2002. Very low to low-grade metamorphic rocks. A proposal on behalf of the IUGS Sub-commission on the Systematic of Metamorphic Rocks.

Aziz, H.A., Adlan, M.N., Ariffin, K.S., 2008. Heavy metals (Cd, Pb, Zn, Ni, Cu and Cr (III)) removal from water in Malaysia: post treatment by high quality limestone. Bioresource Technology, 99, 1578-83.

Bellen, R. C., Dunnington, H. V., Wetzel, R., Morton, D. M., 1959. Lexique Stratigraphy International. Paris, vol. III, Asie, Fascicule 10a, 1-311. 
Bozkaya, O., Yalcin, H., 2000. Very low-grade metamorphism of Upper Paleozoic-Lower Mesozoic sedimentary rocks related to sedimentary burial and thrusting in Central Taurus Belt, Konya, Turkey. International Geology Review, 42(4), 353-367.

Brumsack, H.J., 2006. The trace metal content of recent organic carbon-rich sediments: implications for Cretaceous black shale formation. Paleogeographic. Palaeoclimatology. Palaeoecology., 232, 344-361.

Cardott, B.J., Lambert, M.W., 1985. Thermal maturation by vitrinite reflectance of the Woodford shale, Anadarko Basin, Oklahoma. American Association Petroleum Geology Bulltein, 69, 1982-1988.

Condie, K.C., 1991. Another look at rare earth elements in shales. Geochim. Cosmochim. Acta, 55, $2527-2531$.

Dellisanti, F.G.A., Pini, G.A., Baudin, F., 2010. Use of Tmax as a thermal maturity indicator in orogenic successions and comparison with clay mineral evolution. Clay Mineral, 45, 115-130.

Fu, X., Wang, J., Zeng, Y., Tan, F., Feng, X., 2010. REE geochemistry of marine oil shale from the Changsha Mountain area, northern Tibet, China. International Journal Coal Geology, 81, 191-199.

Fu, X. G, Wang, J., Zeng, Y. H., Tan, F. W., He, J. L., 2011. Geochemistry and origin of rare earth elements (REEs) in the Shengli River oil shale, northern Tibet, China. Chem der Erde, 71, 21-30.

Fu, X., Wang, J., Feng, X., Chen, W., Wang, D., Song, C., Zeng, S., 2017. Mineralogical compositions and elemental enrichment of shales in lacustrine rift basin: a case study in the Cenozoic Lunpola Basin, central Tibet. Geological Journal, 53 (2), 439-457.

Hakimi, M.H, Mohialdeen, I.M.J., Al-Ahmed, A.A., El-Nady, M.M., 2017. Thermal modeling and hydrocarbon generation of the Late Jurassic-Early Cretaceous Chia Gara Formation in Iraqi Kurdistan region, northern Zagros Fold Belt. Egyptian Journal Petroleum, 27(4), 701-713.

Hardy, R., Tucker, M., 1988. X-ray powder diffraction of sediments. M. Tucker, M. (Ed.), Techniques in Sedimentology, Blackwell Science, Cambridge,191-228.

Jassim, S.Z., Goff, C., 2006. Geology of Iraq. Dolin, Prague and Moravian Museum.

Jones, B., Manning, D.A.C., 1994. Composition of geochemical indices used for the interpretation of paleoredox conditions in ancient mudstones. Chemical Geology, 111, 111-129.

Kubler, B., 1964. Les argiles, indicateurs de métamorphisme. Rev Inst. Franch Petrology, 19, 1093-1112.

Kübler, B., Jaboyedoff, F., 2000. Physical meaning and applications of the illite Kübler index: measuring reaction progress in low-grade metamorphism. Earth Planet Science, 331, 75-89.

Liang, L., Morgan, J.J., 1990. Chemical aspects of iron oxides coagulation in water: Laboratory studies and implications for natural systems. Aquatic Science, 52, 32-55.

Lucas, J., Abbas, M. 1989. Uranium in natural phosphorites: The Syrian example. Sciences Geologiques, bulletin. et mém, 42, 223-236.

Luoma, S.N., Rainbow, P.S., 2008. Metal contamination in aquatic environment. Science and lateral management, Cambridge, 1-573.

Mohialdeen, I.M.J., Hakimi, M.H., Al-Beyati, F.M., 2013. Geochemical and petrographic characterization of Late Jurassic-Early Cretaceous Chia Gara Formation in Northern Iraq: Paleoenvironment and oil-generation potential. Marine Petroleum Geology, 43, 166-177.

Moore, D.M., Reynolds, R.C., 1997. X-Ray Diffraction and the identification and analysis of clay minerals. Oxford University Press.

Murris, R.J., 1980. Middle East- Stratigraphic evolution and oil habitat. AAPG Bulltein, 64(5), 597-618.

Mustafa, R.K., Tobia, F.H., 2020. Geochemical application in unraveling paleo-weathering, provenance and environmental setting of the shale from Chia Gara Formation, Kurdistan Region, Iraq. Iraqi Geological Journal, 53, 90-116.

Numan, N.M.S., 1997. A plate tectonic scenario for the Phanerozoic succession in Iraq. Iraqi Geological Journal, $30,1-28$.

Odisho, K.Y., Othman, R.S., 1992. Preliminary geochemical evaluation of hydrocarbon source rocks in northern part of Iraq. Iraqi Geological Journal, 25, 136-153.

Ohr, M., Halliday, A.N., Peacor, D.R., 1994. Mobility and fractionation of rare earth elements in argillaceous sediments: implications for dating diagenesis and low-grade metamorphism. Geochim. Cosmochim. Acta, 58, 289-312. 
Pancost, R.D., Crawford, N., Magness, S., Turner, A., Jenkyns, H.C., Maxwell, J.R., 2004. Further evidence for the development of photic-zone euxinic conditions during Mesozoic oceanic anoxic events, Journal Geological Society, 161, 353-364.

Peacor, D.R., 1992. Diagenesis and low-grade metamorphism of shales and slates. In: Buseck, P.R. (Ed.), Minerals and Reactions at the Atomic Scales: Transmission Electron Microscopy. Reviews in Mineralogy 27. Mineral Society American, 335-380.

Pevear, D.R., 1999. Illite and hydrocarbon exploration. Proceedings of the National Academy of Sciences of the United States of America, 96, 3440-3446.

Rainer, T., Herlec, U., Rantitsch, G., Sachsenhofer, R., Vrabec, M., 2002. Organic matter maturation vs clay mineralogy: a comparison for Carboniferous to Eocene sediments from the Alpine-Dinaride junction (Slovenia, Austria). Geologija, 45, 513-518.

Qing, W., Jingru, B., Jianxin, G.,Yan-Zhen, W., Shuyuan, L., 2014. Geochemistry of rare earth and other trace elements in chinese oil shale. Oil Shale, 31(3), 266-277.

Rakovan, J., Reederr, J., Elzinga, E.J., Cherniak, D., Tait, C.D., Morris, D.E., 2002. Characterization of U(VI) in the apatite structure by X-ray absorption spectroscopy. Environment Science Technology, 36, 3114-3117.

Schultz, R.B., 2004. Geochemical relationships of Late Palaeozoic carbon-rich shales of the Midcontinent, USA: a compendium of results advocating changeable geochemical conditions. Chemistry Geology, 206, 347372.

Segonzac, G.D., 1969. Les mineraux argileux dans la diagene`se. Passage au me 'tamorphisme. Service Carte Geology Alsace-Lorraine, Mem., 29, 1-320.

Sharland, P.R., Archer, R., Casey, D.M., Davies, R.B., Hall, S.H., Heward, A.P., Horbury, A.D., Simmons, M.D., 2001. Arabian Plate Sequence Stratigraphy. In: Geo Arabia, Special Publication 2, 1-372.

Sherwani, G.H., Dilbi, A.N.F., 2019. Microfacies and depositional environment of Chia Gara Formation (Upper Jurassic- Lower Cretaceous) in Kurdistan region, Northern Iraq. Iraqi Bulltein Geology Mining, 15, 31-42.

Silva, P.L., Bustin, R.M., 2020. Significance and Distribution of Apatite in the Triassic Doig Phosphate Zone, Western Canada Sedimentary Basin. Minerals, 10.

Spath, L.F., 1950. A new Tithonian Ammonoid fauna from Kurdistan, North Iraq. Bulletin of the British Museum (Natural History) Geology, 1(4), 95- 135.

Spear, D.A., Zheng, Y., 1999. Geochemistry and origin of elements in some UK coals. International Journal Coal Geology, 38, 161-179.

Sutherland, R.A., 2000. Bed sediment-associated trace metals in an urban stream, Oahu. Hawaii. Environment Geology, 39, 611-627.

Taylor, S.R., McLennan, S.M., 1985. The Continental Crust: Its Composition and Evolution. Blackwell, Oxford, UK, 349 pp.

Tobia, F.H., Al-Jaleel, H.S., Ahmad, I.N., 2018. Provenance and depositional environment of the Middle-Late Jurassic shales, northern Iraq, Geoscience Journal, 23 (5), 747-765.

Tourtelot, A., 1979. Black shale-its deposition and diagenesis. Clays and Clay Miner., 27, 313-321.

Tribovillard, N., Riboulleau, A., Lyons, T., Baudin, F., 2004. Enhanced trapping of molybdenum by sulfurized marine organic matter of marine origin in Mesozoic limestones and shales. Chemistry Geology, 213, 385401.

Whittington, II R.A., 2009. Clay mineralogy and illite crystallinity in the Late Devonian to early Mississippian Woodford shale in the Arbuckle Mountains, Oklahoma, USA, M.Sc. Thesis, Georgia State University, 139 pp.

Wignall, P.B., 1994. Black Shales: Oxford Monographs on Geology and Geophysics, 30. Clarendon Press, Oxford, $127 \mathrm{pp}$.

Wiseman, C.L.S., Püttmann, W., 2006. Interactions between mineral phases in the preservation of soil organic matter. Geoderma, 134(1), 109-118. 